\title{
Some identities in density-functional theory
}

\author{
Robert G. Parr and Shubin Liu \\ Department of Chemistry, University of North Carolina, Chapel Hill, North Carolina 27599
}

Alfred A. Kugler

60 McLeod St. No. 507, Ottawa, Ontario, Canada K2P 261

Á. Nagy

Institute of Theoretical Physics, Kossuth Lajos University, H-4010 Debrecen, Hungary

\author{
(Received 6 December 1994)
}

\begin{abstract}
Various density-functional relations are established involving functional expansions for quantities of interest. The central identity expresses an arbitrary well-behaved functional $F[\rho]$ in terms of the actual density $\rho(\mathbf{x})$ and the functional derivatives of $F[\rho]$ evaluated at the density $\rho(\mathbf{x})$. A number of useful consequences of the formulas are given.
\end{abstract}

PACS number(s): 31.15.Ew, 03.65.Db

\section{INTRODUCTION}

The first part of this paper establishes an identity of a rather general nature and validity for an arbitrary functional $F[\rho]$ in density-functional theory. Here $\rho(x)$ is the electron density in an atom or a molecule, which satisfies the normalization condition

$$
\int d \mathbf{x} \rho(\mathbf{x})=N,
$$

where $N$ is the total number of electrons and the integration extends over all space. Some examples of functionals of interest are the kinetic energy $T[\rho]$, the electronelectron interaction energy $V_{e e}[\rho]$, and the exchangecorrelation energy $E_{\mathrm{xc}}[\rho]$. The second part of this paper develops a number of particularly interesting consequences.

In Ref. [1], starting from the virial theorem, one of the authors derived the following identity for the kineticenergy functional $T_{0}[\rho]$ of an inhomogeneous system of noninteracting fermions:

$$
\begin{aligned}
& T_{0}[\rho]= \int d \mathbf{x} \rho(\mathbf{x}) T_{0}^{\prime}(\mathbf{x} ;[\rho]) \\
&- \frac{1}{2} \iint d \mathbf{x}_{1} d \mathbf{x}_{2} \rho\left(\mathbf{x}_{1}\right) \rho\left(\mathbf{x}_{2}\right) T_{0}^{\prime \prime}\left(\mathbf{x}_{1}, \mathbf{x}_{2} ;[\rho]\right) \\
&+\frac{1}{3 !} \iiint d \mathbf{x}_{1} d \mathbf{x}_{2} d \mathbf{x}_{3} \rho\left(\mathbf{x}_{1}\right) \rho\left(\mathbf{x}_{2}\right) \rho\left(\mathbf{x}_{3}\right) \\
& \times T_{0}^{\prime \prime \prime}\left(\mathbf{x}_{1}, \mathbf{x}_{2}, \mathbf{x}_{3} ;[\rho]\right)-\cdots
\end{aligned}
$$

The primes on the quantities in the foregoing equation denote functional derivatives with respect to the density $\rho$, e.g.,

$$
T_{0}^{\prime \prime}\left(\mathbf{x}_{1}, \mathbf{x}_{2} ;[\rho]\right) \equiv \frac{\delta^{2} T_{0}[\rho]}{\delta \rho\left(\mathbf{x}_{1}\right) \delta \rho\left(\mathbf{x}_{2}\right)} .
$$

If the quantities $T_{0}^{\prime}(\mathbf{x} ;[\rho]), T_{0}^{\prime \prime}\left(\mathbf{x}_{1}, \mathbf{x}_{2} ;[\rho]\right), \ldots$ are known, Eq. (2) can be regarded as a functional expansion of $T_{0}[\rho]$ in terms of the density $\rho(\mathbf{x})$. In Ref. [1] it is also shown that for the homogeneous noninteracting Fermi gas in one and two dimensions, the series expansion (2) terminates after a few terms, thus giving the kinetic energy in terms of the first few functional derivatives.

Functional expansions such as Eq. (2) have been presented in several recent papers [2-4]. In Sec. II we establish that for any general, well-behaved functional $F[\rho]$ an identity or functional expansion such as Eq. (2) holds. In Sec. III we develop a number of consequences. These results are of interest in density-functional theory for two reasons. First, they may reveal interesting connections between quantities of physical interest. Second, they may permit the evaluation of some needed but unknown quantity in terms of other quantities. In particular, they may help in the evaluation of some quantity in terms of its functional derivatives.

\section{GENERAL FUNCTIONAL EXPANSION}

The identity in question is provided by the following. Theorem 1. For any well-behaved function $F[\rho]$,

$$
\begin{aligned}
F[\rho]= & C+\int d \mathbf{x} \rho(\mathbf{x}) F^{\prime}(\mathbf{x} ;[\rho]) \\
- & -\frac{1}{2} \iint d \mathbf{x}_{1} d \mathbf{x}_{2} \rho\left(\mathbf{x}_{1}\right) \rho\left(\mathbf{x}_{2}\right) F^{\prime \prime}\left(\mathbf{x}_{1}, \mathbf{x}_{2} ;[\rho]\right) \\
+ & \frac{1}{3 !} \iiint d \mathbf{x}_{1} d \mathbf{x}_{2} d \mathbf{x}_{3} \rho\left(\mathbf{x}_{1}\right) \rho\left(\mathbf{x}_{2}\right) \rho\left(\mathbf{x}_{3}\right) \\
& \times F^{\prime \prime \prime}\left(\mathbf{x}_{1}, \mathbf{x}_{2}, \mathbf{x}_{3} ;[\rho]\right)-\cdots .
\end{aligned}
$$

Here "well-behaved" means that all of the indicated functional derivatives exist and that the indicated functional series converges, at least in a region near $\rho$. The value of the constant is zero if the region of convergence of the series includes $\rho=0$ and $F[0]=0$. However, there may be cases in which $\rho=0$ is not included in the region of convergence and for which the value of the constant is not zero. See below and the Appendix.

For a preliminary incomplete proof of Eq. (4), consider 
the expansion

$$
\begin{aligned}
F\left[\rho_{2}\right]=F\left[\rho_{1}\right]+\int & d \mathbf{x}\left[\rho_{2}(\mathbf{x})-\rho_{1}(\mathbf{x})\right] F^{\prime}\left(\mathbf{x} ;\left[\rho_{1}\right]\right) \\
+\frac{1}{2} \iint & d \mathbf{x}_{1} d \mathbf{x}_{2}\left[\rho_{2}\left(\mathbf{x}_{1}\right)-\rho_{1}\left(\mathbf{x}_{1}\right)\right] \\
& \times\left[\rho_{2}\left(\mathbf{x}_{2}\right)-\rho_{1}\left(\mathbf{x}_{2}\right)\right] \\
& \times F^{\prime \prime}\left(\mathbf{x}_{1}, \mathbf{x}_{2} ;\left[\rho_{1}\right]\right)+\cdots,
\end{aligned}
$$

where the successive functional derivatives are all evaluated at the density $\rho_{1}$. Taking $\rho_{1}=\rho$ and $\rho_{2}=0$, Eq. (4) is immediately obtained. It is not necessary, however, to view Eq. (4) as an expansion about zero density, as shown in the Appendix. All that is necessary is for the functional derivatives to exist and the series to converge, at the density of interest. One could say that $F[\rho]$ must be "reasonably analytic" at $\rho$. The Appendix gives the proof of Eq. (4) for the case in which $\rho=0$ is not necessarily included in the region of convergence.

Comment 1. The significance of Eq. (4) from a physical point of view is that it provides an expression for the quantity $F[\rho]$ in terms of the actual density $\rho(x)$ and functional derivatives, which can be related to kernels or correlation functions associated with the atomic or molecular system (see, e.g., $[1,2]$ ).

Comment 2. Equation (4) is the functional analog of an identity for functions that can be derived as follows. Suppose

$f(y)=f(x)+(y-x) f^{\prime}(x)+\frac{1}{2}(y-x)^{2} f^{\prime \prime}(x)-\cdots$.

Then it is readily verified upon similarly expanding $f^{\prime}(y), f^{\prime \prime}(y), \ldots$ that

$$
\begin{aligned}
& f(x)-x f^{\prime}(x)+\frac{1}{2} x^{2} f^{\prime \prime}(x)-\cdots \\
& =f(y)-y f^{\prime}(y)+\frac{1}{2} y^{2} f^{\prime \prime}(y)-\cdots=\text { const } .
\end{aligned}
$$

This remark is due to Cedillo [18].

Comment 3. For functionals of physical interest, the region of convergence of Eq. (4) often includes $\rho=0$, with $F(0)=0$. This is the case for the functionals mentioned in the Introduction. In what follows, for convenience, we take the constant in Eq. (4) to be zero.

Comment 4. The validity of Eq. (4) is not dependent on whether or not the functional $F[\rho]$ obeys any simple scaling relation. Thus, while a scaling property can be used to establish Eq. (4) in particular cases, the converse does not hold, i.e., the relation (4) does not imply the existence of a scaling property for $F[\rho]$.

Comment 5. Equation (4) was used in Ref. [2] in connection with an expansion of what was there called the hardness functional.

Comment 6. An example where the expansion (4) terminates after a few terms is provided by the classical Coulomb interaction energy

$$
V_{c}[\rho]=\frac{e^{2}}{2} \iint d \mathbf{x} d \mathbf{x}^{\prime} \frac{\rho(\mathbf{x}) \rho\left(\mathbf{x}^{\prime}\right)}{\left|\mathbf{x}-\mathbf{x}^{\prime}\right|}
$$

In this case

$$
\begin{aligned}
& V_{c}^{\prime}(\mathbf{x} ;[\rho])=\frac{\delta V_{c}[\rho]}{\delta \rho(\mathbf{x})}=e^{2} \int d \mathbf{x}^{\prime} \frac{\rho\left(\mathbf{x}^{\prime}\right)}{\left|\mathbf{x}-\mathbf{x}^{\prime}\right|}, \\
& V_{c}^{\prime \prime}\left(\mathbf{x}, \mathbf{x}^{\prime}\right)=\frac{\delta^{2} V_{c}[\rho]}{\delta \rho(\mathbf{x}) \delta \rho\left(\mathbf{x}^{\prime}\right)}=\frac{e^{2}}{\left|\mathbf{x}-\mathbf{x}^{\prime}\right|}, \\
& V_{c}^{(n)}\left(\mathbf{x}, \mathbf{x}^{\prime}, \mathbf{x}^{\prime \prime}, \ldots\right)=0, \quad n \geq 3
\end{aligned}
$$

and the right-hand side of Eq. (4) becomes

$$
\begin{gathered}
\int d \mathbf{x} \rho(\mathbf{x}) V_{c}^{\prime}(\mathbf{x} ;[\rho])-\frac{1}{2} \iint d \mathbf{x} d \mathbf{x}^{\prime} \rho(\mathbf{x}) \rho\left(\mathbf{x}^{\prime}\right) V_{c}^{\prime \prime}\left(\mathbf{x}, \mathbf{x}^{\prime}\right) \\
=\left(1-\frac{1}{2}\right) e^{2} \iint d \mathbf{x} d \mathbf{x}^{\prime} \frac{\rho(\mathbf{x}) \rho\left(\mathbf{x}^{\prime}\right)}{\left|\mathbf{x}-\mathbf{x}^{\prime}\right|}=V_{c}[\rho] .
\end{gathered}
$$

Comment 7. An important example of the application of Eq. (4) for which the constant is not zero would be the description of a molecular electronic density as a perturbation of superposed atomic densities.

\section{SOME ADDITIONAL IDENTITIES FOR FUNCTIONAL EXPANSIONS}

\section{A. Strictly local functionals}

By a strictly local functional we mean here a functional that can be written as an integral of a function $f$ of $\rho(x)$, i.e.,

$$
F[\rho]=\int d \mathbf{x} f(\rho(\mathbf{x})),
$$

where $f$ does not contain gradient terms or higher-order derivatives of $\rho(x)$. We note that for such a functional, the first few functional derivatives are

$$
\begin{aligned}
& F^{\prime}(\mathbf{x} ;[\rho])=f^{\prime}(\rho(\mathbf{x})), \\
& F^{\prime \prime}\left(\mathbf{x}_{1}, \mathbf{x}_{2} ;[\rho]\right)=f^{\prime \prime}\left(\rho\left(\mathbf{x}_{1}\right)\right) \delta\left(\mathbf{x}_{1}-\mathbf{x}_{2}\right), \\
& \left.F^{\prime \prime \prime}\left(\mathbf{x}_{1}, \mathbf{x}_{2}, \mathbf{x}_{3} ; \rho\right]\right)=f^{\prime \prime \prime}\left(\rho\left(\mathbf{x}_{1}\right)\right) \delta\left(\mathbf{x}_{1}-\mathbf{x}_{2}\right) \delta\left(\mathbf{x}_{1}-\mathbf{x}_{3}\right),
\end{aligned}
$$

where $f^{(n)}(\rho) \equiv d^{n} f(\rho) / d \rho^{n}$. Hence, substituting Eqs. (14)-(16) into Eq. (4), we obtain the functional expansion for functionals satisfying Eq. (13),

$$
F[\rho]=\sum_{n=1}^{\infty} \frac{(-)^{n-1}}{n !} \int d \mathbf{x}[\rho(\mathbf{x})]^{n} f^{(n)}(\rho(\mathbf{x})) .
$$

This type of functional includes the class of homogeneous functionals

$$
F[\rho]=\int d \mathbf{x}[\rho(\mathbf{x})]^{k},
$$

where the index $k$ is a positive number. For such a functional

$$
\int d \mathbf{x} \rho(\mathbf{x}) f^{\prime}(\rho(\mathbf{x}))=k F[\rho]
$$

and the identity (17) is equivalent to

$$
\begin{aligned}
0=\int d \mathbf{x}[\rho(\mathrm{x})]^{k}[ & 1-k+\frac{1}{2} k(k-1) \\
& \left.-\frac{1}{6} k(k-1)(k-2)+\cdots\right] .
\end{aligned}
$$

Equation (20) is obviously correct since the quantity in the second set of square brackets is the binomial expansion for $(1-1)^{k}$. As another example, the reader may easily verify the identity (17) for the functional 


$$
F[\rho]=\int d \mathbf{x} \rho(x) \ln \rho(\mathbf{x}) .
$$

Theorem 2. For the functional defined by Eq. (13), the following identity holds:

$$
F[\rho]=-\frac{1}{3} \int d \mathbf{x} \mathbf{x} \cdot \nabla \rho(\mathbf{x}) f^{\prime}(\rho(\mathbf{x})) .
$$

Proof. Although a shorter proof is indicated below [Eqs. (33) and (34)], we first follow a longer path to show the connection and consistency with the expansion (17). Consider the functional $I_{n}[\rho]$ defined by

$$
I_{n-1}[\rho]=\int d \mathbf{x}[\rho(\mathbf{x})]^{n-1} \mathbf{x} \cdot \nabla \rho(\mathbf{x}) f^{(n)}(\rho(\mathbf{x})),
$$

where $n$ is a positive integer. Integration by parts leads to the identity

$$
\frac{1}{3}\left\{I_{n-1}[\rho]+\frac{1}{n} I_{n}[\rho]\right\}=-\frac{1}{n} \int d \mathbf{x}[\rho(\mathbf{x})]^{n} f^{(n)}(\rho(\mathbf{x})) \text {. }
$$

Multiplying both sides of Eq. (24) by $(-1)^{n-1} /(n-1)$ ! and summing over $n$ from 1 to $\infty$ we notice that on the left-hand side all terms but the first cancel out, leaving only the term on the right-hand side of Eq. (22). The summation on the right-hand side yields precisely the series (17). This establishes the identity (22) for the strictly local functional of Eq. (13). [Note that if the number of spatial dimensions is $d$ rather than 3 , the factor $\frac{1}{3}$ in Eqs. (22) and (24) must be replaced by $1 / d$.]

Comments. For some particular $F[\rho]$ such that $F$ and $\delta F / \delta \rho(\mathbf{x})$ can be calculated, Eq. (22) can be used as a test of whether $F[\rho]$ is strictly local. The identity (22) is a generalization of the one obtained in Ref. [8] for a spherically symmetric system.

Suppose now that the functional $F[\rho]$, in addition to being strictly local, also satisfies a scaling relation of the form

$$
F\left[\rho_{\lambda}\right]=\lambda^{k} F[\rho],
$$

where $\rho_{\lambda}(\mathbf{x})=\lambda^{3} \rho(\lambda \mathbf{x})$ and $\lambda$ is a positive scale factor. It is well known [5-7] that in this case $F[\rho]$ obeys the identity

$$
\begin{aligned}
F[\rho] & =-\frac{1}{k} \int d \mathbf{x} \rho(\mathbf{x}) \mathbf{x} \cdot \nabla \frac{\delta F[\rho]}{\delta \rho(\mathbf{x})} \\
& =\frac{1}{k} \int d \mathbf{x}[3 \rho(\mathbf{x})+\mathbf{x} \cdot \nabla \rho(\mathbf{x})] \frac{\delta F[\rho]}{\delta \rho(\mathbf{x})},
\end{aligned}
$$

where Eq. (27) is obtained from Eq. (26) by integration by parts.

Theorem 3. A strictly local functional that obeys the scaling relation (25) is homogeneous of degree $(1+k / 3)$, i.e,. satisfies the relation

$$
\int d \mathbf{x} \rho(\mathbf{x}) f^{\prime}(\rho(\mathbf{x}))=\left(1+\frac{k}{3}\right) F[\rho] .
$$

Proof. Equation (28) follows directly from Eqs. (14), (22), and (27).

Comments. Equation (28) is well known and forms the basis of the (strictly) local-density approximation (LDA) in density-functional theory $[9,10]$. As examples, for the noninteracting kinetic-energy functional $T_{0}[\rho]$, the scaling index $k=2$, whereas for the exchange (only) energy functional $E_{x}[\rho], k=1$. Equation (28) shows then that $T_{0}[\rho]$ and $E_{x}[\rho]$ are homogeneous functionals of degree $\frac{5}{3}$ and $\frac{4}{3}$ in $\rho(\mathbf{x})$, respectively, i.e., the results of the Thomas-Fermi model and the Dirac exchange model [11].

\section{B. Inclusion of gradient terms}

We now wish to extend the analysis to the case where the functional $F[\rho]$ also depends on the gradient of the local density, i.e., we consider a functional of the form

$$
F[\rho]=\int d \mathbf{x} g(\mathbf{x}, \rho(\mathbf{x}), \nabla \rho(\mathbf{x})),
$$

where $g$ is a function of $\mathbf{x}, \rho(\mathbf{x})$, and $\nabla \rho(\mathbf{x})$, but does not contain higher-order gradients of $\rho(x)$. By allowing $g$ to depend explicitly on the position variable $\mathbf{x}$, the functional (29) also covers the case of the so-called weighteddensity approximation [cf. Eqs. (65)-(73) below].

Theorem 4. For the functional defined by Eq. (29), the following identity holds:

$$
\begin{array}{rl}
F[\rho]+\frac{1}{3} \int d \mathbf{x} & \mathbf{x} \cdot \nabla \rho(\mathbf{x}) \frac{\delta F[\rho]}{\delta \rho(\mathbf{x})} \\
& =\frac{1}{3} \int d \mathbf{x}\left[\rho_{j}(\mathbf{x}) \frac{\partial g}{\partial \rho_{j}(\mathbf{x})}-\mathbf{x} \cdot \frac{\partial g}{\partial \mathbf{x}}\right],
\end{array}
$$

where

$$
\rho_{j}(\mathbf{x}) \equiv \frac{\partial \rho(\mathbf{x})}{\partial x_{j}}
$$

and summation over repeated indices is implied (here and in what follows).

Proof. For the functional (29), the functional derivative is given by

$$
\frac{\delta F[\rho]}{\delta \rho(\mathbf{x})}=\frac{\partial g}{\partial \rho(\mathbf{x})}-\frac{\partial}{\partial x_{i}} \frac{\partial g}{\partial \rho_{i}(\mathbf{x})} .
$$

As is easily seen from an integration by parts, the functional defined by Eq. (29) can also be represented as

$$
F[\rho]=-\frac{1}{3} \int d \mathbf{x} \mathbf{x} \cdot \nabla_{T} g(\mathbf{x}, \rho(\mathbf{x}), \nabla \rho(\mathbf{x})),
$$

where $\nabla_{T}$ indicates the total derivative with respect to $\mathbf{x}$, i.e.,

$$
F[\rho]=-\frac{1}{3} \int d \mathbf{x}\left[\mathbf{x} \cdot \frac{\partial g}{\partial \mathbf{x}}+\mathbf{x} \cdot \nabla \rho \frac{\partial g}{\partial \rho}+\mathbf{x}_{j} \frac{\partial g}{\partial \rho_{i}} \frac{\partial \rho_{i}}{\partial \mathbf{x}_{j}}\right] .
$$

From Eqs. (29), (32), and (34) it follows that

$$
\begin{aligned}
F[\rho] & +\frac{1}{3} \int d \mathbf{x} \mathbf{x} \cdot \nabla \rho(\mathbf{x}) \frac{\delta F[\rho]}{\delta \rho(\mathbf{x})} \\
= & -\frac{1}{3} \int d \mathbf{x}\left\{\mathbf{x} \cdot \frac{\partial g}{\partial \mathbf{x}}+\mathbf{x} \cdot \nabla \rho \frac{\partial}{\partial \mathbf{x}_{i}} \frac{\partial g}{\partial \rho_{i}}+\mathbf{x}_{j} \frac{\partial}{\partial \rho_{i}} \frac{\partial \rho_{i}}{\partial \mathbf{x}_{j}}\right\} .
\end{aligned}
$$


Upon integration by parts, one finds

$$
\begin{aligned}
-\frac{1}{3} \int d \mathbf{x} \times \mathbf{\nabla} \rho \frac{\partial}{\partial \mathbf{x}_{i}} \frac{\partial g}{\partial \rho_{i}}= & \frac{1}{3} \int d \times \delta_{i j} \rho_{j} \frac{\partial g}{\partial \rho_{i}} \\
& +\frac{1}{3} \int d \times \mathbf{x}_{j} \frac{\partial \rho_{j}}{\partial \mathbf{x}_{i}} \frac{\partial g}{\partial \rho_{i}}
\end{aligned}
$$

so that, altogether, the right-hand side of (35) yields

$$
\begin{aligned}
\frac{1}{3} \int d \mathbf{x} \rho_{j} \frac{\partial g}{\partial \rho_{j}}+\frac{1}{3} \int d \mathbf{x} x_{j} \frac{\partial g}{\partial \rho_{i}} & \left.\frac{\partial \rho_{j}}{\partial \mathbf{x}_{i}}-\frac{\partial \rho_{i}}{\partial \mathbf{x}_{j}}\right] \\
& -\frac{1}{3} \int d \mathbf{x} \mathbf{x} \cdot \frac{\partial g}{\partial \mathbf{x}} .
\end{aligned}
$$

Since the middle term in large parentheses of this expression vanishes, we are left with the expression on the right-hand side of Eq. (30). Q.E.D.

Corollary. In most cases of practical interest, the function $g$ depends on the magnitude of the gradient, i.e., on

$|\nabla \rho(\mathbf{x})|=\left[\left(\frac{\partial \rho}{\partial \mathbf{x}}\right)^{2}+\left[\frac{\partial \rho}{\partial y}\right)^{2}+\left(\frac{\partial \rho}{\partial z}\right)^{2}\right]^{1 / 2}$.

In this case, the functional derivative (32) becomes

$$
\frac{\delta F[\rho]}{\delta \rho(\mathbf{x})}=\frac{\partial g}{\partial \rho(\mathbf{x})}-\left[\frac{\partial g}{\partial|\nabla \rho|} \frac{\nabla^{2} \rho}{|\nabla \rho|}+\nabla \rho \cdot \nabla\left[\frac{1}{|\nabla \rho|} \frac{\partial g}{\partial|\nabla \rho|}\right]\right]
$$

and the identity (30) becomes

$$
\begin{aligned}
F[\rho]+\frac{1}{3} \int d \times \mathbf{x} \cdot \nabla \rho(\mathbf{x}) \frac{\delta F[\rho]}{\delta \rho(\mathbf{x})} \\
=\frac{1}{3} \int d \mathbf{x}\left[|\nabla \rho(\mathbf{x})| \frac{\partial g}{\partial|\nabla \rho(\mathbf{x})|}-\mathbf{x} \cdot \frac{\partial g}{\partial \mathbf{x}}\right] .
\end{aligned}
$$

Proof. Equations (39) and (40) follow from Eqs. (32) and (30), respectively, since in this case

$$
\begin{aligned}
\frac{\partial}{\partial x_{i}} \frac{\partial g}{\partial \rho_{i}(\mathbf{x})} & =\frac{\partial}{\partial x_{i}}\left[\frac{\partial g}{\partial|\nabla \rho|} \frac{\partial|\nabla \rho|}{\partial \rho_{i}}\right] \\
& =\frac{\partial}{\partial x_{i}}\left[\frac{\partial g}{\partial|\nabla \rho|} \frac{\rho_{i}}{|\nabla \rho|}\right] \\
& \equiv \nabla \cdot\left[\frac{\nabla \rho}{|\nabla \rho|} \frac{\partial g}{\partial|\nabla \rho|}\right],
\end{aligned}
$$

leading to Eq. (39), while

$\rho_{j} \frac{\partial g}{\partial \rho_{j}}=\rho_{j} \frac{\partial g}{\partial|\nabla \rho|} \frac{\rho_{j}}{|\nabla \rho|}=\frac{\nabla \rho \cdot \nabla \rho}{|\nabla \rho|} \frac{\partial g}{\partial|\nabla \rho|}=|\nabla \rho| \frac{\partial g}{\partial|\nabla \rho|}$,

leading to Eq. (40).

Of particular interest is the case of spherical symmetry. In this case,

$$
\begin{aligned}
& \rho(\mathbf{x})=\rho(r), \\
& \mathbf{x} \cdot \nabla \rho(\mathbf{x})=r \rho^{\prime}(r), \quad r \equiv|\mathbf{x}| \\
& |\nabla \rho(\mathbf{x})|=\rho^{\prime}(r) .
\end{aligned}
$$

The functional derivative (39) becomes the simple expression

$$
\frac{\delta F[\rho]}{\delta \rho(\mathbf{x})}=\frac{\partial g}{\partial \rho(r)}-\left(\frac{2}{r}+\frac{\partial}{\partial r}\right) \frac{\partial g}{\partial \rho^{\prime}(r)}
$$

and the identity (40) becomes

$$
\begin{aligned}
F[\rho]+\frac{4 \pi}{3} \int_{0}^{\infty} d r r^{3} \rho^{\prime}(r) \frac{\delta F[\rho]}{\delta \rho(r)} \\
\quad=\frac{4 \pi}{3} \int_{0}^{\infty} d r r^{2}\left[\rho^{\prime}(r) \frac{\partial g}{\partial \rho^{\prime}(r)}-r \frac{\partial g}{\partial r}\right] .
\end{aligned}
$$

The reader may verify Eq. (45) directly starting from the identity

$$
\begin{aligned}
F[\rho] & =4 \pi \int_{0}^{\infty} d r r^{2} g\left(r, \rho(r), \rho^{\prime}(r)\right) \\
& =-\frac{4 \pi}{3} \int_{0}^{\infty} d r r^{3} \frac{d g}{d r}\left(r, \rho(r), \rho^{\prime}(r)\right),
\end{aligned}
$$

where $d g / d r$ denotes the total derivative with respect to the variable $r$. Equation (46) assumes, of course, that for $r \rightarrow \infty$ and 0 , the function $g$ is such that the limits $r^{3} g I_{0}^{r \rightarrow \infty}$ both vanish.

Comment. Equation (45) also obtains for the spherical average of the functional defined by Eq. (29). Thus, defining the function $\bar{g}\left(r, \rho(r), \rho^{\prime}(r)\right)$ by

$$
\bar{g}\left(r, \rho(r), \rho^{\prime}(r)\right)=\frac{1}{4 \pi} \int d \Omega g(\mathbf{x}, \rho(\mathbf{x}), \nabla \rho(\mathbf{x})),
$$

where the integration is over the solid angle, we find that the functional

$$
\begin{aligned}
F[\rho] & =\int d \times \mathbf{x}(\mathbf{x}, \rho(\mathbf{x}), \nabla \rho(\mathbf{x})) \\
& =4 \pi \int_{0}^{\infty} d r r^{2} \bar{g}\left(r, \rho(r), \rho^{\prime}(r)\right)
\end{aligned}
$$

satisfies Eq. (45).

\section{Generalized gradient approximation}

The foregoing section applies, in particular, to the socalled generalized gradient approximation (GGA) method, which has been applied in recent years in particular to the exchange (only) functional $E_{x}[\rho][12-16]$. In the GGA (also referred to as the gradient expansion method up to second order), functionals of interest are of the form

$$
F[\rho]=\int d \mathbf{x} g(\rho(\mathbf{x}),|\nabla \rho(\mathbf{x})|) .
$$

Note that here the dependence of $g$ on $\mathbf{x}$ is only through $\rho(\mathbf{x})$ and $|\nabla \rho(\mathbf{x})|$; hence the last term on the right-hand side of Eq. (40) is absent and the latter becomes

$$
\begin{aligned}
F[\rho]+\frac{1}{3} \int d \mathbf{x} \mathbf{x} \cdot \nabla \rho(\mathbf{x}) & \frac{\delta F[\rho]}{\delta \rho(\mathbf{x})} \\
= & \frac{1}{3} \int d \mathbf{x}|\nabla \rho(\mathbf{x})| \frac{\partial g}{\partial|\nabla \rho(\mathbf{x})|} .
\end{aligned}
$$

Comments. In the absence of gradient terms in the function $g$, the right-hand side of Eq. (50) is zero, thus reproducing the identity (22) of the LDA, Also, Eq. (50) 
provides a criterion and test of the extent to which the GGA is, or is not, satisfied for a given functional $F[\rho]$.

Let us now assume that $F[\rho]$ also satisfies the scaling or virial relations Eqs. (25)-(27). In this case, when these are combined with Eq. (50), we find the relation

$$
\begin{aligned}
F^{\mathrm{GGA}}[\rho]= & \frac{1}{k} \int d \mathbf{x}[3 \rho(\mathbf{x})+\mathbf{x} \cdot \nabla \rho(\mathbf{x})] v_{F}^{\mathrm{GGA}}(\mathbf{x} ;[\rho]) \\
= & \frac{3}{k+3} \int d \mathbf{x} \rho(\mathbf{x}) v_{F}^{\mathrm{GGA}}(\mathbf{x} ;[\rho]) \\
& +\frac{1}{k+3} \int d \mathbf{x}|\nabla \rho(\mathbf{x})| \frac{\partial g}{\partial|\nabla \rho(\mathbf{x})|}
\end{aligned}
$$

where

$$
\begin{aligned}
{\left[v_{F}^{\mathrm{GGA}}(\mathbf{x} ;[\rho])\right.} & \left.\equiv \frac{\delta F^{\mathrm{GGA}}[\rho]}{\delta \rho(\mathbf{x})}\right] \\
& =\frac{\partial g}{\partial \rho(\mathbf{x})}-\nabla \cdot\left[\frac{\nabla \rho(\mathbf{x})}{|\boldsymbol{\nabla} \rho(\mathbf{x})|} \frac{\partial g}{\partial|\nabla \rho(\mathbf{x})|}\right] .
\end{aligned}
$$

Equation (51b) represents the generalization of Eq. (28) in the GGA. When the expression (52b) is substituted in Eq. (51b), we find that the latter can be reexpressed (after integration by parts) as

$$
\begin{aligned}
F^{\mathrm{GGA}}[\rho]= & \frac{3}{k+3} \int d \mathbf{x} \rho(\mathbf{x}) \frac{\partial g}{\partial \rho(\mathbf{x})} \\
& +\frac{4}{k+3} \int d \mathbf{x}|\nabla \rho(\mathbf{x})| \frac{\partial g}{\partial|\nabla \rho(\mathbf{x})|} .
\end{aligned}
$$

Equation (53) provides a simple and precise method to compare the relative contributions of (strictly) local and gradient terms to the value of the functional $F^{\mathrm{GGA}}[\rho]$.

The two most prominent examples to which Eqs. (49)-(53) apply are the exchange-energy functional $E_{x}[\rho]$ and the (noninteracting) kinetic-energy functional $T_{0}[\rho]$. In the GGA, $E_{x}[\rho]$ is written as

$$
E_{X}^{\mathrm{GGA}}[\rho]=\int d \mathbf{x} e_{X}(\rho(\mathbf{x}),|\nabla \rho(\mathbf{x})|),
$$

where $e_{x}$ denotes the exchange-energy density. This expression must satisfy Eq. (50). The so-called exchange potential $v_{x}(\mathbf{x} ;[\rho])$ is the functional derivative

$$
v_{x}(\mathbf{x} ;[\rho])=\frac{\delta E_{x}[\rho]}{\delta \rho(\mathbf{x})} .
$$

$E_{x}[\rho]$ and $v_{x}(\mathbf{x} ;[\rho])$ are related via the scaling relations with the index $k=1$; explicitly $[5,6]$

$$
E_{x}[\rho]=-\int d \mathbf{x} \rho(\mathbf{x}) \mathbf{x} \cdot \nabla v_{x}(\mathbf{x} ;[\rho]) .
$$

In the GGA, $v_{x}(\mathbf{x} ;[\rho])$ must be evaluated via Eq. (52b) with $e_{x}(\rho,|\nabla \rho|)$ substituted for $g$. We then find from Eqs. (51)-(53) the following equivalent relations for $E_{x}^{\mathrm{GGA}}[\rho]$ :

$$
\begin{aligned}
E_{x}^{\mathrm{GGA}}[\rho] & =\int d \mathbf{x}[3 \rho(\mathbf{x})+\mathbf{x} \nabla \rho(\mathbf{x})] v_{x}^{\mathrm{GGA}}(\mathbf{x} ;[\rho]) \\
& =\frac{3}{4} \int d \mathbf{x} \rho(\mathbf{x}) v_{x}^{\mathrm{GGA}}(\mathbf{x} ;[\rho])+\frac{1}{4} \int d \mathbf{x}|\nabla \rho(x)| \frac{\partial e_{x}}{\partial|\nabla \rho(\mathbf{x})|} \\
& =\frac{3}{4} \int d \mathbf{x} \rho(\mathbf{x}) \frac{\partial e_{x}}{\partial \rho(\mathbf{x})}+\int d \mathbf{x}|\nabla \rho(\mathbf{x})| \frac{\partial e_{x}}{\partial|\nabla \rho(\mathbf{x})|} .
\end{aligned}
$$

The (noninteracting) kinetic-energy functional $T_{0}[\rho]$ obeys the scaling relations $(25)-(27)$ with the index $k=2$; explicitly $[5,6,1]$

$$
T_{0}[\rho]=-\frac{1}{2} \int d \mathbf{x} \rho(\mathbf{x}) \mathbf{x} \cdot \nabla \frac{\delta T_{0}[\rho]}{\partial \rho(\mathbf{x})} .
$$

In the GGA, $T_{0}[\rho]$ is written as

$$
T_{0}^{\mathrm{GGA}}[\rho]=\int d \mathbf{x} t_{0}(\rho(\mathbf{x}),|\nabla \rho(\mathbf{x})|),
$$

where $t_{0}$ denotes the kinetic-energy density. The expression (59) must satisfy the identity (50). Also

$$
\frac{\delta T_{0}^{\mathrm{GGA}}[\rho]}{\delta \rho(\mathbf{x})}=\frac{\partial t_{0}}{\partial \rho(\mathbf{x})}-\nabla \cdot\left[\frac{\nabla \rho(\mathbf{x})}{|\nabla \rho(\mathbf{x})|} \frac{\partial t_{0}}{\partial|\nabla \rho(\mathbf{x})|}\right]
$$

In this case, Eqs. (51)-(53) yield the following equivalent expressions for $T_{0}^{\mathrm{GGA}}[\rho]$ :

$$
\begin{aligned}
T_{0}^{\mathrm{GGA}}[\rho] & =\frac{3}{5} \int d \mathbf{x} \rho(\mathbf{x}) \frac{\delta T_{0}^{\mathrm{GGA}}[\rho]}{\delta \rho(\mathbf{x})}+\frac{1}{5} \int d \mathbf{x}|\nabla \rho(\mathbf{x})| \frac{\partial t_{0}}{\partial|\nabla \rho(\mathbf{x})|} \\
& =\frac{3}{5} \int d \mathbf{x} \rho(\mathbf{x}) \frac{\partial t_{0}}{\partial \rho(\mathbf{x})}+\frac{4}{5} \int d \mathbf{x}|\nabla \rho(\mathbf{x})| \frac{\partial t_{0}}{\partial|\nabla \rho(\mathbf{x})|} \\
& =\frac{4}{5} \int d \mathbf{x} \rho(\mathbf{x}) \frac{\delta T_{0}^{\mathrm{GGA}}[\rho]}{\delta \rho(\mathbf{x})}-\frac{1}{5} \int d \mathbf{x} \rho(\mathbf{x}) \frac{\partial t_{0}}{\partial \rho(\mathbf{x})}
\end{aligned}
$$


The most frequently encountered functional $T_{0}[\rho]$ in the GGA is the Thomas-Fermi-Weizsäcker (TFW) functional (see, e.g., [1] and references therein) in which $t_{0} \mid(\rho,|\nabla \rho|)$ is taken as

$t_{\mathrm{TFW}}(\rho,|\nabla \rho|)=\frac{\hbar^{2}}{m}\left[a[\rho(\mathbf{x})]^{5 / 3}+b \frac{|\nabla \rho(\mathbf{x})|^{2}}{\rho(\mathbf{x})}\right]$,

where $a$ and $b$ are constants. For this functional one has, using Eq. (60),

$$
\begin{aligned}
\frac{\delta T_{\mathrm{TFW}}[\rho]}{\delta \rho(\mathbf{x})}=\frac{\hbar^{2}}{m} & \left(a \frac{5}{3}[\rho(\mathbf{x})]^{2 / 3}\right. \\
& \left.+b\left[\left|\frac{\nabla \rho(\mathbf{x})}{\rho(\mathbf{x})}\right|^{2}-2 \frac{\nabla^{2} \rho(\mathbf{x})}{\rho(\mathbf{x})}\right]\right]
\end{aligned}
$$

and the reader may easily verify that Eqs. (61a) and (61b) are satisfied by $T_{\text {TFW }}[\rho]$.

\section{Weighted-density approximation}

In the weighted-density approximation (WDA), the functional $F[\rho]$ is expressed as

$$
F^{\mathrm{WDA}}[\rho]=\int d \times \mathbf{x} g(\mathbf{x}, \rho(\mathbf{x})),
$$

i.e., there is an explicit dependence of $g$ on the position variable $\mathbf{x}$ in addition to dependence on $\rho(x)$. The WDA is obtained as a special case of Eq. (30); in this case the latter gives

$$
\begin{aligned}
F^{\mathrm{WDA}}[\rho]+\frac{1}{3} \int d \mathbf{x} \mathbf{x} \cdot \nabla \rho(\mathbf{x}) \frac{\delta F^{\mathrm{WDA}}[\rho]}{\delta \rho(\mathbf{x})} \\
=-\frac{1}{3} \int d \mathbf{x} \mathbf{x} \cdot \frac{\partial g}{\partial \mathbf{x}},
\end{aligned}
$$

where now

$$
\frac{\delta F^{\mathrm{WDA}}[\rho]}{\delta \rho(\mathbf{x})}=\frac{\partial g}{\partial \rho(\mathbf{x})} .
$$

When combined with the scaling relation (27) we obtain the equivalent identities

$$
\begin{aligned}
F^{\mathrm{WDA}}[\rho] & =\frac{1}{k} \int d \mathbf{x}[3 \rho(\mathbf{x})+\mathbf{x} \cdot \nabla \rho(\mathbf{x})] \frac{\partial g}{\partial \rho(\mathbf{x})} \\
& =\frac{3}{k+3} \int d \mathbf{x} \rho(\mathbf{x}) \frac{\partial g}{\partial \rho(\mathbf{x})}-\frac{1}{k+3} \int d \mathbf{x} \mathbf{x} \cdot \frac{\partial g}{\partial \mathbf{x}} .
\end{aligned}
$$

In the case of spherical symmetry, Eqs. (67a) and (67b) become

$$
\begin{aligned}
F^{\mathrm{WDA}}[\rho]= & \frac{4 \pi}{k} \int_{0}^{\infty} d r r^{2}\left[3 \rho(r)+r \rho^{\prime}(r)\right] \frac{\partial g}{\partial \rho(r)} \\
= & \frac{3}{k+3} 4 \pi \int_{0}^{\infty} d r r^{2} \rho(r) \frac{\partial g}{\partial \rho(r)} \\
& -\frac{1}{k+3} 4 \pi \int_{0}^{\infty} d r r^{3} \frac{\partial g}{\partial r} .
\end{aligned}
$$

Applying Eqs. (67) to the exchange energy $(k=1)$ we have, in the WDA,

$$
\begin{aligned}
E_{x}^{\mathrm{WDA}}[\rho] & =\int d \mathbf{x}[3 \rho(\mathbf{x})+\mathbf{x} \cdot \nabla \rho(\mathbf{x})] \frac{\partial e_{x}}{\partial \rho(\mathbf{x})} \\
& =\frac{3}{4} \int d \mathbf{x} \rho(x) \frac{\partial e_{x}}{\partial \rho(\mathbf{x})}-\frac{1}{4} \int d \mathbf{x} \mathbf{x} \cdot \frac{\partial e_{x}}{\partial \mathbf{x}} .
\end{aligned}
$$

For the (noninteracting) kinetic energy $(k=2)$, Eqs. (67) become

$$
\begin{aligned}
T_{0}^{\mathrm{WDA}}[\rho] & =\frac{1}{2} \int d \mathbf{x}[3 \rho(\mathbf{x})+\mathbf{x} \cdot \nabla \rho(\mathbf{x})] \frac{\partial t_{0}}{\partial \rho(\mathbf{x})} \\
& =\frac{3}{5} \int d \mathbf{x} \rho(\mathbf{x}) \frac{\partial t_{0}}{\partial \rho(\mathbf{x})}-\frac{1}{5} \int d \mathbf{x} \mathbf{x} \cdot \frac{\partial t_{0}}{\partial \mathbf{x}}
\end{aligned}
$$

As the simplest example of $E_{x}^{\mathrm{WDA}}[\rho]$, consider the functional [3]

$$
E_{x}^{\mathrm{WDA}}[\rho]=-e^{2} \int d \mathbf{x} \rho(\mathbf{x}) \frac{1}{|\mathbf{x}|}, e_{x}=-e^{2} \frac{\rho(\mathbf{x})}{|\mathbf{x}|}
$$

It is easily seen that this functional satisfies Eqs. (69a) and (69b). Moreover, the associated exchange potential $v_{x}^{\text {WDA }}$ is given by

$$
v_{x}^{\mathrm{WDA}}(\mathbf{x})=\frac{\delta E_{x}^{\mathrm{WDA}}}{\delta \rho(\mathbf{x})}=-\frac{e^{2}}{|\mathbf{x}|}
$$

and is independent of $\rho(x)$. In fact, the expression (72) represents the exact exchange potential $v_{x}(x ;[\rho])$ in the asymptotic limit $|\mathbf{x}| \rightarrow \infty$ [17].

\section{CONCLUDING REMARKS}

In his paper we have derived and discussed various identities relating to functionals of interest in densityfunctional theory and their expansion in terms of the density $\rho(x)$. For the most general functional $F[\rho]$, we have obtained an expansion Eq. (4), which expresses $F[\rho]$ in terms of the densities at various points in space without invoking any "reference density" $\rho_{0}(\mathbf{x})$ about which $F[\rho]$ is expanded. The cost for doing so lies in the (generally unknown) $n$-point functions or kernels $F^{(n)}\left(\mathbf{x}_{1}, \ldots, \mathbf{x}_{n} ;[\rho]\right)$, which are the functional derivatives of $F[\rho]$ evaluated at the actual density $\rho(x)$.

We have shown the connection and consistency of the general functional expansion Eq. (4) to that obtained from Eq. (13) if $F[\rho]$ is, or is approximated by, a strictly local functional (LDA). In this case, the functional expansion reduces to a very simple identity Eq. (17). Going beyond the LDA to include gradient terms in $F[\rho]$, we have obtained a number of identities relating $F[\rho]$ to $\delta F[\rho] / \delta \rho(\mathbf{x})$. In particular, we have considered the generalized gradient approximation from a general point of view and have found a number of relations that must be satisfied regardless of the specific form chosen to express $F[\rho]$.

These relations should serve to illuminate the general aspects underlying various calculations from the functional point of view.

\section{ACKNOWLEDGMENTS}

This research has been aided by grants from the $\mathrm{Na}$ tional Science Foundation, the Petroleum Research Fund 
of the American Chemical Society, the Exxon Education Foundation, and the Hungarian-U.S. Science and Technology Joint Fund.

\section{APPENDIX: DERIVATION OF EQ. (4)}

Suppose the functional $F[\rho]$ is such that it allows a functional Taylor expansion about some density $\rho_{1}(\mathbf{x})$. In particular, this implies the existence of functional derivatives of $F[\rho]$ of all orders in the function space surrounding the points (in function space) being considered. Define

$$
\Delta \rho(\mathbf{x})=\rho_{2}(\mathbf{x})-\rho_{1}(\mathbf{x}) .
$$

Then we have via the Taylor expansion about $\rho(x)$

$$
\begin{aligned}
\Delta F \equiv & F\left[\rho_{2}\right]-F\left[\rho_{1}\right] \\
= & \left.\int d \mathbf{x} \frac{\delta F[\rho]}{\delta \rho(\mathbf{x})}\right|_{\rho=\rho_{1}} \Delta \rho(\mathbf{x}) \\
& +\left.\frac{1}{2} \iint d \mathbf{x}_{1} d \mathbf{x}_{2} \frac{\delta^{2} F[\rho]}{\delta \rho\left(\mathbf{x}_{1}\right) \delta \rho\left(\mathbf{x}_{2}\right)}\right|_{\rho=\rho_{1}} \Delta \rho\left(\mathbf{x}_{1}\right) \Delta \rho\left(\mathbf{x}_{2}\right) \\
& +\frac{1}{3 !} \iiint \cdots .
\end{aligned}
$$

For brevity we set

$$
\begin{aligned}
& \left.F_{1}^{\prime} \equiv \frac{\delta F[\rho]}{\delta \rho(\mathbf{x})}\right|_{\rho=\rho_{1}(x)},\left.\quad F_{2}^{\prime} \equiv \frac{\delta F[\rho]}{\delta \rho(\mathbf{x})}\right|_{\rho=\rho_{2}(\mathbf{x})}, \\
& \left.F_{1}^{\prime \prime} \equiv \frac{\delta^{2} F[\rho]}{\delta \rho(\mathbf{x}) \delta \rho\left(\mathbf{x}^{\prime}\right)}\right|_{\rho=\rho_{1}(\mathbf{x})}, \\
& \left.F_{2}^{\prime \prime} \equiv \frac{\delta^{2} F[\rho]}{\delta \rho(\mathbf{x}) \delta \rho\left(\mathbf{x}^{\prime}\right)}\right|_{\rho=\rho_{2}(\mathbf{x})},
\end{aligned}
$$

etc. For the subsequent development it is important to note that the above functional derivatives are symmetric with respect to interchange of any pair of arguments. We rewrite (A2) as

$$
\begin{aligned}
\Delta F= & F_{2}-F_{1} \\
= & \int F_{1}^{\prime}\left(\rho_{2}-\rho_{1}\right)+\frac{1}{2} \iint F_{1}^{\prime \prime}\left(\rho_{2}-\rho_{1}\right)\left(\rho_{2}-\rho_{1}\right) \\
& +\frac{1}{3 !} \iiint F_{1}^{\prime \prime \prime}\left(\rho_{2}-\rho_{1}\right)\left(\rho_{2}-\rho_{1}\right)\left(\rho_{2}-\rho_{1}\right)+\cdots,
\end{aligned}
$$

where the notation has been abbreviated. We also expand the quantities $F_{2}^{\prime}, F_{2}^{\prime \prime}, \ldots$ about $\rho=\rho_{1}(\mathbf{x})$. Thus

$$
\begin{aligned}
\Delta F^{\prime}=F_{2}^{\prime}-F_{1}^{\prime}= & \int F_{1}^{\prime \prime}\left(\rho_{2}-\rho_{1}\right) \\
& +\frac{1}{2} \iint F_{1}^{\prime \prime \prime}\left(\rho_{2}-\rho_{1}\right)\left(\rho_{2}-\rho_{1}\right) \\
& +\frac{1}{3 !} \iiint \cdots, \\
\Delta F^{\prime \prime} \equiv F_{2}^{\prime \prime}-F_{1}^{\prime \prime}= & \int F_{1}^{\prime \prime \prime}\left(\rho_{2}-\rho_{1}\right) \\
& +\frac{1}{2} \iint F_{1}^{(I V)}\left(\rho_{2}-\rho_{1}\right)\left(\rho_{2}-\rho_{1}\right) \\
& +\frac{1}{3 !} \iiint \cdots,
\end{aligned}
$$

etc. Equivalently,

$$
\begin{aligned}
F_{1}^{\prime}= & F_{2}^{\prime}-\int F_{1}^{\prime \prime}\left(\rho_{2}-\rho_{1}\right) \\
& -\frac{1}{2} \iint F_{1}^{\prime \prime \prime}\left(\rho_{2}-\rho_{1}\right)\left(\rho_{2}-\rho_{1}\right)-\frac{1}{3 !} \iiint \cdots, \\
F_{1}^{\prime \prime}= & F_{2}^{\prime \prime}-\int F_{1}^{\prime \prime \prime}\left(\rho_{2}-\rho_{1}\right) \\
& -\frac{1}{2} \iint F_{1}^{(I V)}\left(\rho_{2}-\rho_{1}\right)\left(\rho_{2}-\rho_{1}\right)-\frac{1}{3 !} \iiint \cdots .
\end{aligned}
$$

Using (A8), the term $\int F_{1}^{\prime}\left(\rho_{2}-\rho_{1}\right)$ in (A5) can be rewritten as

$$
\begin{aligned}
\int F_{1}^{\prime}\left(\rho_{2}-\rho_{1}\right)= & -\int F_{1}^{\prime} \rho_{1}+\int F_{2}^{\prime} \rho_{2} \\
& -\iint F_{1}^{\prime \prime} \rho_{2}\left(\rho_{2}-\rho_{1}\right) \\
& -\frac{1}{2} \iiint F_{1}^{\prime \prime \prime} \rho_{2}\left(\rho_{2}-\rho_{1}\right)\left(\rho_{2}-\rho_{1}\right) \\
& -\frac{1}{3 !} \iiint \cdots .
\end{aligned}
$$

Substituting (A10) into (A5), the latter becomes

$$
\begin{aligned}
\Delta\left[F-\int F^{\prime} \rho\right]= & \frac{1}{2} \iint F_{1}^{\prime \prime}\left[\left(\rho_{2}-\rho_{1}\right)\left(\rho_{2}-\rho_{1}\right)-2 \rho_{2}\left(\rho_{2}-\rho_{1}\right)\right] \\
& +\frac{1}{3 !} \iiint F_{1}^{\prime \prime \prime}\left[\left(\rho_{2}-\rho_{1}\right)\left(\rho_{2}-\rho_{1}\right)\left(\rho_{2}-\rho_{1}\right)-3 \rho_{2}\left(\rho_{2}-\rho_{1}\right)\left(\rho_{2}-\rho_{1}\right)\right]+\frac{1}{4 !} \iiint \cdots
\end{aligned}
$$

where

$$
\Delta\left[F-\int F^{\prime} \rho\right] \equiv\left[F[\rho]-\int \rho F^{\prime}\right]_{\rho=\rho_{1}}^{\rho=\rho_{2}}
$$

Using the symmetry of $F_{1}^{\prime \prime}$ with resect to its arguments, the first term on the right-hand side of (A11) can be expressed as 


$$
\frac{1}{2} \iint F_{1}^{\prime \prime}\left[\left(\rho_{2}-\rho_{1}\right)\left(\rho_{2}-\rho_{1}\right)-2 \rho_{2}\left(\rho_{2}-\rho_{1}\right)\right]=\frac{1}{2} \iint F_{1}^{\prime \prime}\left(\rho_{1} \rho_{1}-\rho_{2} \rho_{2}\right)
$$

Using (A7), the right-hand side of (A13) can be written as

$$
\frac{1}{2} \iint F_{1}^{\prime \prime} \rho_{1} \rho_{1}-\frac{1}{2}\left(\iint F_{2}^{\prime \prime} \rho_{2} \rho_{2}-\iiint F_{1}^{\prime \prime \prime} \rho_{2} \rho_{2}\left(\rho_{2}-\rho_{1}\right)-\frac{1}{2} \iiint \int \cdots\right) \text {. }
$$

With (A13) and (A14), (A11) becomes

$$
\begin{aligned}
\Delta\left[F-\int F^{\prime} \rho\right]= & \frac{1}{2} \iint F_{1}^{\prime \prime} \rho_{1} \rho_{1}-\frac{1}{2} \iint F_{2}^{\prime \prime} \rho_{2} \rho_{2} \\
& +\frac{1}{3 !} \iiint F_{1}^{\prime \prime \prime}\left[\left(\rho_{2}-\rho_{1}\right)\left(\rho_{2}-\rho_{1}\right)\left(\rho_{2}-\rho_{1}\right)-3 \rho_{2}\left(\rho_{2}-\rho_{1}\right)\left(\rho_{2}-\rho_{1}\right)\right. \\
& \left.+3 \rho_{2} \rho_{2}\left(\rho_{2}-\rho_{1}\right)\right]+\frac{1}{4 !} \iiint \int F_{1}^{(I V)} \ldots \\
= & \frac{1}{2} \iint F_{1}^{\prime \prime} \rho_{1} \rho_{1}-\frac{1}{2} \iint F_{2}^{\prime \prime} \rho_{2} \rho_{2}+\frac{1}{3 !} \iiint F_{1}^{\prime \prime \prime}\left(\rho_{2} \rho_{2} \rho_{2}-\rho_{1} \rho_{1} \rho_{1}\right)+\frac{1}{4 !} \iiint \int F_{1}^{(I V)} \ldots .
\end{aligned}
$$

The pattern is now clear and may be continued in such a way that the quantity $F_{1}^{(n)}$ becomes multiplied by $\rho_{1} \rho_{1} \cdots \rho_{1}$, from which is subtracted the quantity $F_{2}^{(n)}$ multiplied by $\rho_{2} \rho_{2} \cdots \rho_{2}$. We thus arrive at the result

$$
\Delta\left[F-\int F^{\prime} \rho\right]=\Delta\left[-\frac{1}{2} \iint F^{\prime \prime} \rho \rho+\frac{1}{3 !} \iiint F^{\prime \prime \prime \prime} \rho \rho \rho-\frac{1}{4 !} \iiint \int \cdots\right],
$$

where the notation (A12) is used. Apart from a possible constant term (see comment 3 in Sec. II), (A16) is the same as Eq. (4) in the text.

[1] A. A. Kugler, Phys. Rev. A 41, 3489 (1990).

[2] R. G. Parr and J. L. Gázquez, J. Phys. Chem. 97, 3939 (1993).

[3] Á. Nagy, Phys. Rev. A 47, 2715 (1993).

[4] Á. Nagy (unpublished).

[5] S. K. Ghosh and R. G. Parr, J. Chem. Phys. 82, 3307 (1985).

[6] M. Levy and J. P. Perdew, Phys. Rev. A 32, 2010 (1985).

[7] H. Ou-Yang and M. Levy, Phys. Rev. A 44, 54 (1991).

[8] Q. Zhao, R. C. Morrison, and R. G. Parr, Phys. Rev. A 50, 2138 (1994).

[9] P. Hohenberg and W. Kohn, Phys. Rev. 136, B864 (1964).

[10] W. Kohn and L. J. Sham, Phys. Rev. 140, A1133 (1965).

[11] See, for example, H. A. Bethe and R. Jackiw, Intermediate Quantum Mechanics, 3rd ed. (Benjamin/Cummings, Men- lo Park, CA, 1986), Chap. 5; N. H. March, Self-Consistent Fields in Atoms (Pergamon, Oxford, 1975), Chaps. 3 and 7.

[12] J. P. Perdew and Y. Wang, Phys. Rev. B 33, 8800 (1986).

[13] A.D. Becke, J. Chem. Phys. 84, 4524 (1986); Phys. Rev. A 38, 3098 (1988).

[14] A. E. DePristo and J. D. Kress, J. Chem. Phys. 86, 1425 (1987).

[15] S. H. Vosko and L. D. Macdonald, in Condensed Matter Theories, edited by P. Vashishta, R. K. Kali, and R. F. Bishop (Plenum, New York, 1987), Vol. 2, p. 101.

[16] E. Engel and S. H. Vosko, Phys. Rev. B 47, 13164 (1993).

[17] J. D. Talman and W. F. Shadwick, Phys. Rev. A 14, 36 (1976).

[18] A. Cedillo (private communication). 